\section{Automatic Acquisition and Image Analysis of 2D Crystals}

N. Coudray ${ }^{\star}$, F. Beck ${ }^{* *}$, J.-L. Buessler*, A. Korinek*, A. Karathanou ${ }^{\star}$, H. Rémigy $y^{\star * *}$, H. Kihl ${ }^{\star}$, A. Engel ${ }^{\star * *}$, J.M. Plitzko**, J.-P. Urban*

${ }^{\star}$ MIPS Laboratory, Université de Haute Alsace, Mulhouse, France ${ }^{*}$ MPIB, Max Planck Institute of Biochemistry, Molecular Structural Biology, Martinsried, Germany

${ }^{* * * B I O Z E N T R U M, ~ M . E . ~ M u e l l e r ~ I n s t i t u t e ~ f o r ~ S t r u c t u r a l ~ B i o l o g y, ~}$ University of Basel, Switzerland nicolas.coudray@uha.fr

2D-crystallization in combination with transmission electron microscopy (TEM) is one of the few methods for the structural analysis of membrane proteins in their native state. However, the parameters for the generation of large crystalline sheets are typically difficult to identify for a given protein. Many repetitive and time consuming screening steps by TEM are therefore necessary to find the best crystallization and preparation conditions $[1,2]$. Although several software packages offer the possibility to control an electron microscope, none is completely adapted for a fully automated Magnification and completely integrated acquisition and analysis of $2 \mathrm{D}$ crystals. Here we report on the development of a fully automatic screening and on-line analysis $\overline{-\bar{M} \text { edium }}-\bar{?}$ software for the fast and automatic survey of large Magnification quantities of negatively stained EM samples for 2D crystallography.

The software system designed to achieve our objectives is built of two parts: the computer control of the TEM and the on-line image characterization. The control of the microscope (Tecnai F30 'Polara', FEI Company, Eindhoven, The Netherlands, equipped with a Gatan Imaging Filter and a 2048x2048 CCD camera, $30 \mu \mathrm{m}$ pixel size) is based on the acquisition of images using the TOM toolbox, a home-made software package utilizing the scientific computing platform Matlab (The MathWorks, Natick, USA) [3]. The image processing tools create an on-line analysis of the images to characterize the sample. An optimized screening process is guided by the analysis outcome simulating the decision of a microscopist. During this process the crystallinity of the sample is evaluated. Figure 1 shows the interplay of the two parts during the three-step process described below. Crystal quality assessment can be done directly using the diffraction mode of the microscope or by calculating power spectra of images at high magnification. However, screening at high magnification is very inefficient since the abundance of suitable $2 \mathrm{D}$ crystals can be low and the field of view (FOV) at that magnification (typically less than $\left.1 \mu \mathrm{m}^{2}\right)$ is small compared to the entire grid dimensions $(\varnothing \sim 3 \mathrm{~mm}$ ) and the maximal FOV of $\sim 2 \mathrm{~mm}^{2}$. Therefore, two intermediate steps at lower magnifications are required for the complete automation process for the grid quality assessment and the identification of suitable crystals (a segmentation process of images at medium magnification is presented in [4]).

First, at very low magnification (e.g. $175 \times$ ), an area as large as covering $1.96 \mathrm{~mm}^{2}$ of the grid is screened and the resulting map is processed for an initial quality evaluation (i.e. broken carbon film). The position of the suitable mesh squares are extracted by a local histogram analysis method and stored within the program. This step

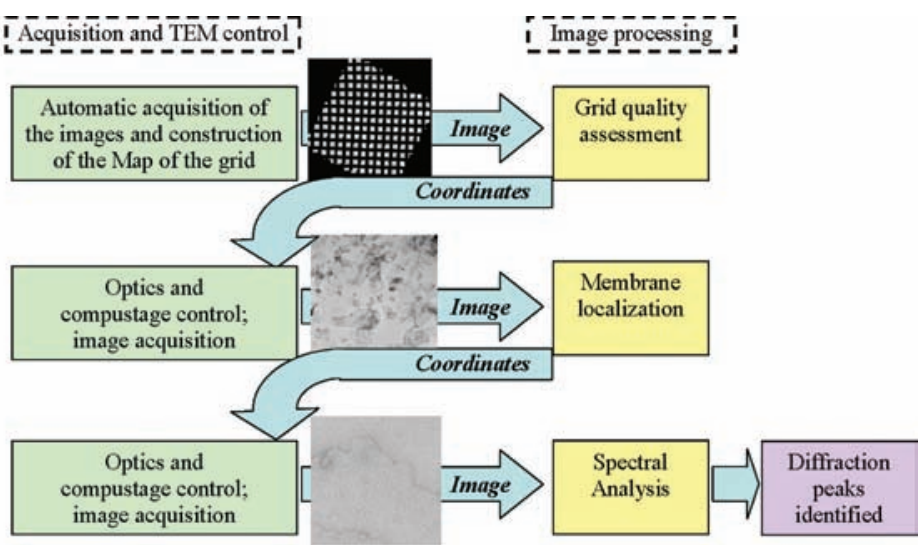

Fig. 1: Automatic control of a TEM for 2D-crystallization evaluation. The diagram describes the interaction between acquisition and TEM control and the image processing tools during the three-step process.
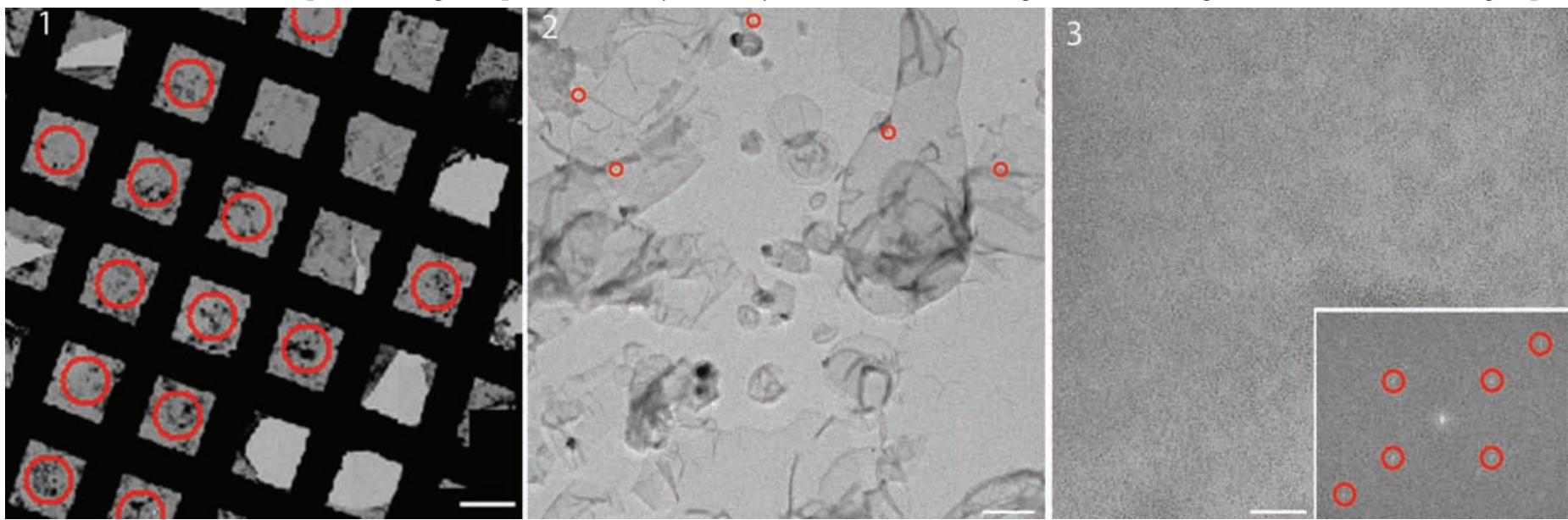

Fig 2: Automated screening micrographs. (1) Map of the grid at low magnification with circles showing the selected squares (scale bar: $20 \mu \mathrm{m}$ ). (2) Image of crystal sheets at medium magnification (scale bar: $1 \mu \mathrm{m}$ ). Circles indicate potential areas for further analysis at higher magnification. (3) High magnified image (scale bar: $10 \mathrm{~nm}$ ) of such an area and corresponding power spectrum (inset) with the identified diffraction peaks (circles).

is essential to avoid spending too much time in further acquisitions on regions where there is no information: on the opaque copper grid, and on regions where the carbon film upon which the specimen is placed is damaged. The microscope is then directed to the determined locations for a further analysis at medium magnification (e.g. 3,000x). After automatic adjustments (auto-focusing, auto-eucentric height) at this magnification, several images per 
grid-square are acquired and analyzed with an edge-based algorithm to identify potentially crystallized areas. Finally, micrographs of these areas are acquired at higher magnification (e.g. 50,000x). To unmistakably identify crystals and to validate the success of the crystallization approach, power spectra of the images are computed for a subsequent peak extraction.

Experiments have been conducted on SoPIP2;1, a plant aquaporin recombinantely expressed in Pichia pastoris [5], and the results are displayed in Figure 2. Results validate the ability of this method to identify crystal in a fully automatic manner.

\section{Acknowledgments}

This work was supported by the EU 6th framework (HT3DEM, LSHG-CT-2005-018811). It is part of the High Throughput Three Dimensional Electron Microscopy (HT3DEM) project set up for the development of an automated platform to enable high throughput production, screening and analysis of $2 \mathrm{D}$ membrane protein crystals (www.ht3dem.org).

\section{References:}

[1] H.W. Rémigy, et al., FEBS Letters 555/1 (2003) 160-169.

[2] M.J. Ellis, H. Hebert, Micron 32/5 (2001) 541-550.

[3] S. Nickell, et al., Journal of Structural Biology 149/3 (2005) 227-234.

[4] N. Coudray, et al., Physics in Signal and Image Processing (PSIP), Mulhouse, France, (2007).

[5] M. Karlsson, et al., FEBS Letters 537 (2003) 68-72.

[6] Acknowledgment: This work was supported by the EU 6th framework (HT3DEM, LSHG-CT-2005-018811).

\section{Customer}

\section{Support}

\section{Engineer}

The NanoAnalysis Division of Oxford Instruments America, Inc. is currently seeking a Field Support Engineer to support our customers. In this role you will perform product installations, customer training, emergency breakdown service and preventative maintenance.

The following describes the ideal candidate:

2 Year Microscopy or Materials Science degrees or equivalent experience.

Specific Experience with Scanning Electron Microscopy X-ray Microanalysis and PC-based instrumentation.

Excels with working within a Windows environment and familiar with Microsoft Office.

Experience/ willingness to travel throughout North America.

Pro-active logical thinker. Customer focused.

High level of organization and ability to document service work

Excellent communication skills with customers, sales representatives,

OEM service engineers and co-workers

- Ability to lift up to $100 \mathrm{lbs}$ with assistance.

Oxford Instruments is a high technology tool and systems business. It has a broad range of capabilities, which provide the tools, processes, and solutions needed to advance fundamental nanoscience research and its transfer into commercial nanotechnology applications. With a unique set of technologies to enable the manipulation and observation of matter at the smallest scales, Oxford Instruments offers solutions for the fabrication and characterization of nanoscale materials, structures and devices, and environments in which to perform fundamental nanoscience. Visit our website at www.oxfordinstruments.com. We offer a comprehensive compensation and benefits package including vacation pay, medical and dental coverage, and a matching $401 \mathrm{k}$ plan. Please submit your resume with salary history to: oxford_nanoafse@ yahoo.com EEO/AA

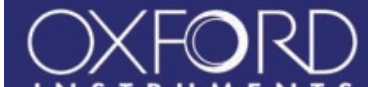

I NSTR U M E T S

The Business of Science ${ }^{\mathrm{TM}}$

\section{Polishing Matters!}

\section{Flat Lapping \& Polishing}
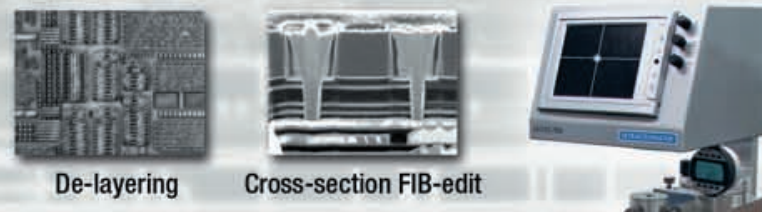

- Optical alignment makes flatness set-up

fast \& accurate

- Wide range of sample holders

- All polishing methods

ULTRAPOL Advance

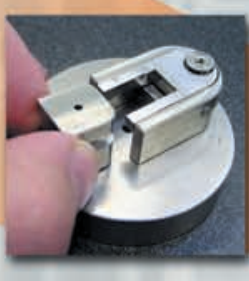

SEM \& FIB STUB HOLDERS

Available for most microscopes

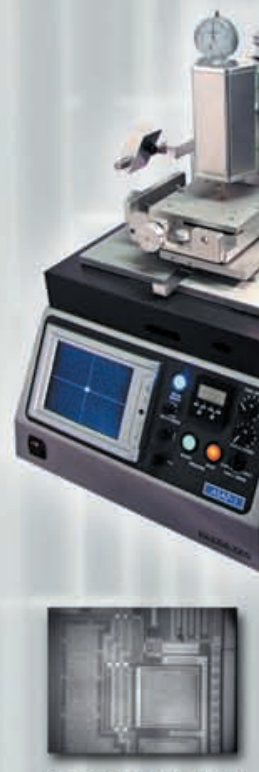

Backside Polishing

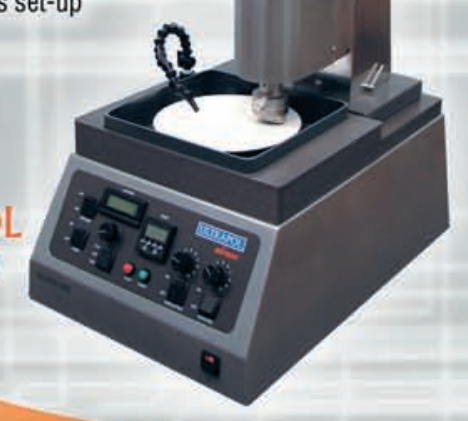

\section{Selected Area} Preparation

- Cavity milling - leaves outside circuits \& structures intact

- Optical alignment

- Decap, grind, lap, \& polish with one machine

\section{ASAP-}

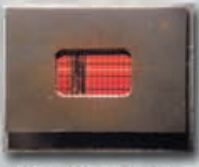

Decapsulation

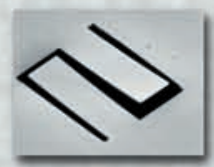

Thin Films (Courtesy of FIBICS, Inc.)
U⿰月, TRA TIEEC MANUFACTURING. INC.

Toll Free (US) 1-877-542-0609

Tel: 1-714-542-0608 Fax: 1-714-542-0627

E-mail: info@ultratecusa.com www.ultratecusa.com 